\title{
One-Step Thermal Processing of BaCe0.8Y0.2O3- $\delta$ Hydrogen Permeable Multi-Channel Hollow Fiber Membrane
}

\author{
Tianlei Wang ${ }^{1}$, Zheng Fan ${ }^{2}$, Shoufei Wang ${ }^{2}$, Qiankun Zheng ${ }^{1}$, Jinkun Tan ${ }^{1}$, Zhengkun Liu ${ }^{2}$, \\ Guangru Zhang ${ }^{1}$, and Wanqin Jin $^{3}$
}

${ }^{1}$ Affiliation not available

${ }^{2}$ Nanjing Tech University

${ }^{3}$ Nanjing University of Technology

September 25, 2021

\begin{abstract}
Yttrium-doped barium cerate $\left(\mathrm{BaCe}_{0.8} \mathrm{Y}_{0.2} \mathrm{O}_{3-\delta}, \mathrm{BCY}\right)$ is the most widely studied proton conducting material and is frequently fabricated as dense membranes for hydrogen separation. However, the difficulty to prepare dense BCY membranes is the extremely high sintering temperature, normally higher than 1500 oC. Herein, the BCY 7-channel hollow fiber membrane was prepared by one-step thermal processing (OSTP). It proved that adding $\mathrm{Co}_{2} \mathrm{O}_{3}$ as sintering aid is beneficial to the densification and $1 \mathrm{wt} \% \mathrm{Co}_{2} \mathrm{O}_{3}$ was the optimum addition to form a homogeneous phase structure. The dense sintering temperature was greatly reduced from over 1500 to $1350{ }^{\circ} \mathrm{C}$. The hydrogen permeation flux of the BCY hollow fiber membrane reached up to $0.34 \mathrm{ml} \mathrm{min}{ }^{-1} \mathrm{~cm}^{-2}$ at $900{ }^{\circ} \mathrm{C}$. The long-term stability test last for $300 \mathrm{~h}$. The properties of OSTP samples were demonstrated to be essentially higher than samples made by conventional ceramic hollow fiber fabrication methods.
\end{abstract}

\section{Hosted file}

AIChE manuscript 0823-fianl.docx available at https://authorea.com/users/436199/articles/ 538700-one-step-thermal-processing-of-bace0-8y0-2o3-\%CE\%B4-hydrogen-permeable-multichannel-hollow-fiber-membrane 